\title{
Creating Coordination in the Cerebellum
}

\author{
Catania, 2-4 October 2003
}

\author{
Pharmacology of the metabotropic GluR-mediated current \\ at the climbing fiber to Purkinje cell synapse \\ PR Andjus, ${ }^{1,2} \mathrm{~L} \mathrm{Zhu}^{1}$ and P Strata ${ }^{1}$ \\ ${ }^{1}$ Department for Neuroscience, University of Turin, Italy, and \\ ${ }^{2}$ Department of Physiology and Biochemistry, School of \\ Biology, University of Belgrade, Serbia E Montenegro
}

Different forms of synaptic plasticity in the cerebellum are mediated by metabotropic glutamate receptors (mGluRs). At parallel fiber (PF) to Purkinje cell (PC) synapses activation of $\mathrm{mGluR}$ gives rise to a well known slow synaptic current inhibited by antagonists of mGluR1. The distribution of mGluR types in the climbing fiber (CF) to PC synapses is not well known. Only recently an mGluR1-mediated all-or-none postsynaptic current was also demonstrated at the CF-PC synapse (Dzubay \& Otis, Neuron 2002; 36: 1159).

Using whole cell patch-clamp recordings from PCs in rat cerebellar slices with AMPA receptors blocked (NBQX, 10 or CNQX, $20 \mu \mathrm{M}$ ) and impaired glutamate uptake (TBOA, $100 \mu \mathrm{M}$ ) we demonstrate a more complex pharmacology of a current obtained by single or train $(10$ or $100 \mathrm{~Hz})$ CF stimulation. The mGluR1 specific antagonist CPCCOEt, $100 \mu \mathrm{M}$ in a group of cells suppressed this response while in a similar number of other cells it induced a potentiating effect. The antagonists of mGluR groups II and III (LY341495 and MSOP, respectively) predominantly suppressed the current. In addition, $100 \mu \mathrm{M}$ MSOP did not occlude the inhibition by $0.2 \mu \mathrm{M}$ LY341495.

The ambiguous effect of CPCCOEt was checked by measuring the paired-pulse depression of the CF EPSC, which was not changed with the antagonist. The pairedpulse plasticity was also not changed by CPCCOEt in low $(0.5 \mathrm{mM})$ external $\mathrm{Ca}^{2+}$ (used to prevent saturation of AMPARs), thus excluding a presynaptic effect. However, CPCCOEt induced a rise in the amplitude (by $\sim 25 \%$ ) as well as a prolongation of the decay time of CF EPSCs at normal $2 \mathrm{mM} \mathrm{Ca}^{2+}$, i.e. under conditions of AMPAR saturation $(11.7 \pm 0.7 \mathrm{~ms}$ vs. $15.8 \pm 1.5 \mathrm{~ms})$, thus indicating an effect of postsynaptic origin. In $0.5 \mathrm{mM} \mathrm{Ca}^{2+}$ the decay of CF EPSCs was faster $(7.5 \pm 1.2 \mathrm{~ms})$ but it was also prolonged by CPCCOEt $(8.8 \pm 1.2 \mathrm{~ms})$. However, the CF EPSC amplitude was not significantly affected indicating an underlying $\mathrm{Ca}^{2+}$-dependent mechanism.

Thus, the pharmacology of the PC mGluR-mediated response points to a dual postsynaptic role of mGluR1 giving rise to a slow postsynaptic current but also regulating other presumably mGluR-dependent currents via second messenger molecules and $\mathrm{Ca}^{2+}{ }_{\mathrm{i}}$. The addi- tional electrophysiological role of mGluR II \& III types was also indicated. Such a complex regulatory mechanism may have an important role in the mGluR-dependent forms of homosynaptic plasticity and motor learning at the CF-PC synapse.

\section{Electrophysiological and optical recording studies of responses induced by stimulation of the inferior olive and parallel fibers in the cerebellum-pons-medulla preparation isolated from neonatal rats}

\author{
A Arata and $M$ Ito \\ Laboratory for Memory and Learning, Brain Science \\ Institute, Riken, Wako Saitama, Japan
}

The cerebellum-pons-medulla preparation isolated from neonatal rat is a unique in vitro preparation that preserves intact neuronal networks including the inferior olive nucleus, pontine nuclei and cerebellar nuclei. This preparation is not only useful for pharmacological approaches, but also advantageous for analyzing the activity of cerebellar cells, keeping neuronal connections to the outside of the cerebellum. Moreover, using optical imaging method, we can identify the responded area projecting from/to stimulation site. In this study, we examined the synaptic responses of Purkinje cells induced by stimulation of inferior olive nucleus/parallel fibers using whole cell patch clamp method and optical imaging.

Cerebellum, pons and medulla were removed from 0to 8-days-old rats, the preparations were cut rostrally as including the cerebellum and pons and cut caudally at the level of the rostral roots of hypoglossal nerves. The isolated preparation was put caudal surface up in the chamber and superfused with modified Krebs solution equilibrated with $95 \% \quad \mathrm{O}_{2}$ and $5 \% \quad \mathrm{CO}_{2}, 26-27^{\circ} \mathrm{C}$. Stimulation electrodes were put into the inferior olive nucleus and on the surface of cerebellar cortex softly. The neurons responding to both inferior olive nucleus (IO) and parallel fibers (PF) stimulation were considered Purkinje cell, and were recorded intracellularly using whole cell patch clamp method. The recorded neurons were stained with Lucifer Yellow and identified their locations and morphology. For optical imaging, we used MiCAM01 system which was developed by Dr Ichikawa and colleague (Laboratory for Brain-Operative Device, Brainway Group, RIKEN). Isolated cerebellum-ponsmedulla preparation from neonatal rat was stained with a voltage sensitive dye (Di-4 ANEPPS or Di-2 ANEPEQ, $0.2 \mathrm{mg} / \mathrm{ml}$ ) for $30 \mathrm{~min}$. Then the preparation was put into the recording chamber and fluorescence changes corre- 
sponding to membrane potential changes induced by stimulation of IO and PF were measured.

In 5-day-old rat cerebellum, the amplitude of PFevoked EPSPs decreased after $1 \mathrm{~Hz}$-conjunctive stimulation of IO and $\mathrm{PF}$ for $5 \mathrm{~min}$, and this depression continued for over an hour. This long-term depression (LTD) was observed in the lateral edge of developing cerebellum. Moreover, optical imaging using voltage sensitive dye revealed that IO stimulation induced strong excitation in the lateral edge of cerebellum (flocculus, paraflocculus, Crus I, Crus II) and PF stimulation was followed by signal propagation along parallel fibers. The optical signal induced by IO stimulation was blocked by non-NMDA-receptor antagonist CNQX $(10 \mu \mathrm{M})$ and was not blocked by NMDA-receptor antagonist APV $(10 \mu \mathrm{M})$. While, the optical signal induced by $\mathrm{PF}$ stimulation was partially blocked by $10 \mu \mathrm{M} \mathrm{APV}$, and mainly blocked by $10 \mu \mathrm{M}$ CNQX. Moreover, optical signal at the site receiving both IO and PF stimulation was depressed over an hour after conjunctive stimulation.

The results suggested that (1) the Purkinje cells that responded to both $\mathrm{IO}$ and $\mathrm{PF}$ stimulation were found in P5 rat cerebellum, (2) analysis of optical signals indicated that the excitation induced by IO stimulation involved AMPA receptors, but the excitation induced by $\mathrm{PF}$ stimulation involved mainly AMPA receptors and partially NMDA receptors, and (3) LTD was observed at the site receiving both $\mathrm{IO}$ and $\mathrm{PF}$ stimulation using whole cell patch clamp method and optical imaging as early as 5 days postnatal in developing cerebellum.

\section{Selective modification of the expression of calretinin into the cerebellar granule cells of transgenic mice}

B Bearzatto, ${ }^{1}$ L Servais, ${ }^{1,3}$ F Baba-Aïssa, ${ }^{1}$ A de Kerchove d'Exaerde, ${ }^{1}$ S Schurmans, ${ }^{2}$ G Cheron ${ }^{3}$ and SN

Schiffmann ${ }^{1}$

${ }^{1}$ Laboratory of Neurophysiology, ${ }^{2}$ IRIBHM CP601

Université Libre de Bruxelles Campus Erasme, Brussels, and ${ }^{3}$ Laboratory of Neurophysiology, Université Mons-Hainaut, Mons, Belgium.

Variations in intracellular $\mathrm{Ca}^{2+}$ concentration $\left(\left[\mathrm{Ca}^{2+}\right] \mathrm{i}\right)$ regulate a large variety of neuronal functions. The control of $\left[\mathrm{Ca}^{2+}\right] \mathrm{i}$ is crucial for the neuron and is performed by pumps, exchangers, organelle sequestration and cytoplasmic $\mathrm{Ca}^{2+}$ binding proteins. Among these $\mathrm{Ca}^{2+}$ binding proteins, calbindin $(\mathrm{Cb})$ and calretinin $(\mathrm{Cr})$ are enriched in the cerebellum: $\mathrm{Cb}$ in Purkinje cells and $\mathrm{Cr}$ in granule, unipolar brush and lugaro cells. Changes in $\left[\mathrm{Ca}^{2+}\right] \mathrm{i}$ in cerebellar neurons would be expected to dramatically affect information processing in the cerebellum and disturb the entire cerebellar physiology. Indeed, analysis of calretinin-deficient $(\mathrm{Cr}-/-)$ mice showed impaired coordination, detection of an unexpected calretinin-like immunoreactivity in Purkinje cells and marked abnormalities in the Purkinje cell firing in vivo.
The absence of $\mathrm{Cr}$ in cerebellar granule cells constitute a main hypothesis consistent with all these perturbations. To investigate this hypothesis, we specifically rescued the expression of $\mathrm{Cr}$ in the cerebellar granule cells of $\mathrm{Cr}-/-$ mice and also generated mice that over-express $\mathrm{Cr}$ in these granule cells. The $\mathrm{Cr}$ expression was targeted to cerebellar granule cells by using a fragment of the gene coding for the GABAA alpha 6 subunit encompassing the promoter and the exons 1 to 8 . This part of the gene has been previously shown to allow restricted transgene expression in cerebellar granule cells. We obtained several lines of transgenic $\mathrm{Cr}-/-$ mice exhibiting a selective and restricted re-expression of $\mathrm{Cr}$ in granule cells as demonstrated by in situ hybridization and immunohistochemistry. These mutant mice will now allow us to assess for the precise role(s) of $\mathrm{Cr}$ in the cerebellar physiology and to elucidate the mechanisms leading to its dysfunction in the $\mathrm{Cr}-/-$ mice. We also believe that, together with other mutants, our model, which allows to alter $\left[\mathrm{Ca}^{2+}\right]$ i homeostasis at specific synapses, will unmask new and unknown aspects of the cerebellar circuitry.

\section{Parallel fiber beams produce a strong excitatory effect on Purkinje cells along the beam only under direct electrical stimulation}

\section{JM Bower ${ }^{1}$ and A Devor ${ }^{2}$ \\ ${ }^{1}$ The Research Imaging Center at The University of Texas Health Science Center, San Antonio and the Cajal Neuroscience Research Center at the University of Texas, San Antonio, Texas, and ${ }^{2}$ NMR Center, Massachusetts General Hospital, Harvard Medical School, Charlestown, Massachusetts, USA}

Many theories of cerebellar function assume that parallel fibers provide strong excitatory input to Purkinje cells situated along the beam, an assumption supported by physiological studies in which parallel fibers are directly activated electrically. However, those studies in which cerebellar circuitry is activated by peripheral somatosensory stimulation have failed to demonstrate beam-like patterns of activation. Instead, this type of stimulation results in patches of activated Purkinje cells that directly overlie activated regions of the granule cell layer. In the present study, we have used reflectance optical imaging to directly contrast direct electrical stimulation of the parallel fibers with peripheral tactile stimulation of the upper leap in vivo in the same animal. Optical imaging of intrinsic signals was combined with extracellular electrophysiological recordings from Purkinje and granule cells. Electrical stimulation of the parallel fibers generated a beam of hemodynamic response aligned with the direction of parallel fibers, and simultaneous electrophysiological recordings showed clear Purkinje cell responses along the beam. In contrast, tactile stimulation of the upper leap produced patches of hemodynamic activity 